\title{
Polyhedral results for the edge capacity polytope
}

\author{
Citation for published version (APA):
}

van Hoesel, C. P. M., Koster, A. M. C. A., van de Leensel, R. L. J. M., \& Savelsbergh, M. W. P. (2002). Polyhedral results for the edge capacity polytope. Mathematical Programming, 92(2), 335-358. https://doi.org/10.1007/s101070200292

Document status and date:

Published: 01/01/2002

DOI:

10.1007/s101070200292

Document Version:

Publisher's PDF, also known as Version of record

\section{Please check the document version of this publication:}

- A submitted manuscript is the version of the article upon submission and before peer-review. There can be important differences between the submitted version and the official published version of record.

People interested in the research are advised to contact the author for the final version of the publication, or visit the DOI to the publisher's website.

- The final author version and the galley proof are versions of the publication after peer review.

- The final published version features the final layout of the paper including the volume, issue and page numbers.

Link to publication

\footnotetext{
General rights rights.

- You may freely distribute the URL identifying the publication in the public portal. please follow below link for the End User Agreement:

www.umlib.nl/taverne-license

Take down policy

If you believe that this document breaches copyright please contact us at:

repository@maastrichtuniversity.nl

providing details and we will investigate your claim.
}

Copyright and moral rights for the publications made accessible in the public portal are retained by the authors and/or other copyright owners and it is a condition of accessing publications that users recognise and abide by the legal requirements associated with these

- Users may download and print one copy of any publication from the public portal for the purpose of private study or research.

- You may not further distribute the material or use it for any profit-making activity or commercial gain

If the publication is distributed under the terms of Article $25 \mathrm{fa}$ of the Dutch Copyright Act, indicated by the "Taverne" license above, 


\title{
Polyhedral results for the edge capacity polytope
}

\author{
Received: September 2000 / Accepted: October 2001 \\ Published online March 27, 2002 - (c) Springer-Verlag 2002
}

\begin{abstract}
Network loading problems occur in the design of telecommunication networks, in many different settings. For instance, bifurcated or non-bifurcated routing (also called splittable and unsplittable) can be considered. In most settings, the same polyhedral structures return. A better understanding of these structures therefore can have a major impact on the tractability of polyhedral-guided solution methods. In this paper, we investigate the polytopes of the problem restricted to one arc/edge of the network (the undirected/directed edge capacity problem) for the non-bifurcated routing case.

As an example, one of the basic variants of network loading is described, including an integer linear programming formulation. As the edge capacity problems are relaxations of this network loading problem, their polytopes are intimately related. We give conditions under which the inequalities of the edge capacity polytopes define facets of the network loading polytope. We describe classes of strong valid inequalities for the edge capacity polytopes, and we derive conditions under which these constraints define facets. For the diverse classes the complexity of lifting projected variables is stated.

The derived inequalities are tested on (i) the edge capacity problem itself and (ii) the described variant of the network loading problem. The results show that the inequalities substantially reduce the number of nodes needed in a branch-and-cut approach. Moreover, they show the importance of the edge subproblem for solving network loading problems.
\end{abstract}

Key words. network design - mixed integer programming - cutting planes - knapsack with integer capacity

\section{Introduction}

The network loading problem (NLP) occurs in telecommunications problems where demand for capacity for multiple commodities is to be realized by inserting capacity into a given network. The capacity can be placed in different sizes, usually multiples of each other. We restrict ourselves to a single capacity size, although many of the ideas presented in this paper can be extended in case multiple capacity sizes

C.P.M. van Hoesel: Dept. of Quantitative Economics, Maastricht University, P.O. Box 616, 6200 MD Maastricht, The Netherlands

R.L.M.J. van de Leensel: CQM B.V., P.O. Box 414, 5600 AK Eindhoven, The Netherlands

The research was carried out while this author was a Ph.D. student at Maastricht University

A.M.C.A. Koster: Konrad-Zuse-Zentrum für Informationstechnik Berlin, Takustraße 7, D-14195 Berlin, Germany, e-mail: koster@zib.de

Corresponding author. The research was partly carried out while this author was a Ph.D. student at Maastricht University

M.W.P. Savelsbergh: School of Industrial and Systems Engineering, Georgia Institute of Technology, Atlanta, GA 30332-0205, USA 
are available. Along with a capacity plan a routing of all commodities is to be determined to obtain a capacity plan that facilitates the demands of all commodities. This problem has been studied in many variants with respect to network lay-out, capacity usage, and routing possibilities. Routing of the demand can be done by reserving capacity on a subnetwork that consists of a single path between the endpoints of a commodity only (non-bifurcated routing), or of a set of paths (bifurcated routing). The cases are also known as unsplittable and splittable flow, respectively. We only consider the non-bifurcated case which is also studied by Gavish and Altinkemer [11] and Brockmüller et al. [8,9]. For the bifurcated case we refer to Magnanti et al. $[18,19]$. With respect to capacity usage one can distinguish unidirectional and bidirectional capacity usage, i.e., if an edge contains a unit of capacity this unit can either be used in one or in both directions of the edge. In most studies the unidirectional (or undirected) case is studied. Exceptions are Bienstock and Günlük [7] and Bienstock et al. [6], who study the bidirectional case. Most of our results can be applied to both capacity models; for the sake of clarity, we restrict us to the bidirectional case in this paper (see [15] for the other case). With respect to network lay-out we do not specialize us. For instance, we do not take reliability requirements into account. For the design of survivable networks with bifurcated routing we refer to Wessäly [23] and the references therein, for non-bifurcated routing to Van de Leensel [17]. Also generalizations like network design with multicast commodities are not considered (cf. Bienstock and Bley [5]). However, this paper considers the relaxation of NLP to a single edge of the network, the so-called edge capacity problem (ECP). We are specifically interested in the polyhedral structure of the polytope of ECP as it is an important substructure of all network loading models.

The edge capacity polytope with a single capacity type can be viewed as a $0-1$ knapsack problem with a single integer variable representing the capacity of the knapsack. The closely related knapsack problem with a single continuous capacity variable is studied by Marchand and Wolsey [20]. They employ valid inequalities of the standard 0-1 knapsack problem (see Balas [2], Hammer et al. [14], Wolsey [24]) to obtain valid inequalities for the extended model by projection and lifting. The edge capacity polytope itself has also been studied by Brockmüller et al. [8,9], who derive valid and facet defining inequalities. Magnanti et al. [18] study the version of the polytope in which the binary variables are relaxed to real variables and derive a complete description of the corresponding polytope. Recently, Atamtürk and Rajan [1] study both the edge capacity polytope and its relaxation to real variables.

In this paper we derive various new results for the edge capacity polytope. In Sect. 2, we discuss one of the basic variants of the non-bifurcated network loading problem. A possible integer linear programming formulation is derived, formulation and modeling alternatives are discussed, and the dimension and trivial facets are stated. Next, in Sect. 3, we introduce two polytopes obtained by restricting the network loading problem to a single edge. The polytopes differ in the number of capacity constraints taking into account (one or two). We show that every non-trivial facet defining inequality for the directed edge capacity polytope defines a facet of the overall network loading polytope as well. For the undirected version conditions are derived under which the same result holds. 
The sequel of the paper is devoted to a polyhedral study of both edge capacity polytopes. First, in Sect. 4, the undirected edge capacity polytope is studied. General properties of facet defining inequalities are derived and two classes of valid inequalities are introduced, both generalizing the $c$-strong inequalities of Brockmüller et al. [8]. These classes of inequalities are defined on a projection of the original polytope. We show that the general properties allow to lift the projected variables in polynomial time. Next, in Sect. 5, the directed edge capacity polytope is studied. We introduce for this polytope one class of valid inequalities and derive conditions under which these inequalities define facets. Moreover, we prove that the lifting of valid inequalities for this polytope is $\mathcal{N} \mathcal{P}$-complete in general.

The third part of the paper is devoted to a computational analysis of the effectiveness of the derived inequalities for both the edge capacity polytope itself and the described variant of the network loading problem. In Sect. 6, we discuss the separation of the valid inequalities, computational results for generated edge capacity instances, and the effectiveness of different strategies in solving real-life network loading problems. The paper is closed by some concluding remarks.

\section{Network loading problems}

\subsection{Problem description}

Let $G=(V, E)$ be an undirected connected graph with node set $V$ and edge set $E$. We define the arc set $A$, which contains two directed $\operatorname{arcs}(i, j)$ and $(j, i)$ for every edge $e=\{i, j\} \in E$. Let $Q$ be a set of commodities. Each element $q \in Q$ is a triple $\left(s^{q}, t^{q}, d^{q}\right)$, with $s^{q}, t^{q} \in V, s^{q} \neq t^{q}$, representing a commodity with demand size $d^{q} \in \mathbb{Z}_{0}^{+}$that must be routed from source node $s^{q}$ to sink node $t^{q}$ on a single path through the network. To route a set of commodities on an arc, sufficient capacity must be available. The capacity on an edge is determined by the number of capacity units installed, where each unit has a base capacity $\lambda \in \mathbb{Z}_{0}^{+}$. Depending on the transmission technology, the installed capacity is available in both directions once (bidirectional capacity installation), or has to be shared by the commodities routed across $(i, j)$ and $(j, i)$ (undirected capacity). Bidirectional capacity installation is sometimes simply called directed, since the capacity can be used in both directions. The goal is to minimize the costs of the installed capacity in the network while ensuring that all commodities can be routed from source to sink simultaneously.

\subsection{An integer linear programming formulation}

To formulate the bidirectional network loading problem as an integer program, let $x_{i j} \in \mathbb{Z}_{0}^{+}$be the number of capacity units installed on edge $\{i, j\}$, and let $f_{i j}^{q}$ be a binary variable indicating whether the commodity $q \in Q$ is routed via $\operatorname{arc}(i, j) \in A$ or not. If $c_{i j}$ represents the costs per base capacity unit on edge $\{i, j\} \in E$, then the model reads: 


$$
\begin{aligned}
& \min \sum_{\{i, j\} \in E} c_{i j} x_{i j} \\
& \text { s.t. } \quad \sum_{\{i, j\} \in E} f_{i j}^{q}-\sum_{\{j, i\} \in E} f_{j i}^{q}=\left\{\begin{array}{rl}
1 & \text { if } i=s^{q} \\
-1 & \text { if } i=t^{q} \\
0 & \text { otherwise }
\end{array} \quad \forall q \in Q, \forall i \in V\right. \\
& \lambda x_{i j} \geq \sum_{q \in Q} d^{q} f_{i j}^{q} \quad \forall\{i, j\} \in E \\
& \lambda x_{i j} \geq \sum_{q \in Q} d^{q} f_{j i}^{q} \quad \forall\{i, j\} \in E \\
& f_{i j}^{q}, f_{j i}^{q} \in\{0,1\}, x_{i j} \in \mathbb{Z}_{0}^{+} \quad \forall q \in Q, \forall\{i, j\} \in E .
\end{aligned}
$$

This model is called the Directed Non-bifurcated Flow Model, and the corresponding set of feasible solutions is denoted DNFM. The capacity on an edge is (bi)directed because installed capacity can be used twice, once in each direction. Hence, the required capacity on an edge is determined by the maximum of forward flow and backward flow on the edge. It is called non-bifurcated since the demand of a commodity has to be routed on a single path (i.e. the demand cannot be bifurcated). Finally, flow variables $f_{i j}^{q}$ on individual arcs $(i, j)$ are used to model the routing of a commodity from source node $s^{q}$ to sink node $t^{q}$.

\subsection{Formulation and model alternatives}

The problem can alternatively be formulated with so-called path variables instead of the flow variables, see [15]. Depending on the exact application and level of aggregation, other variants of the problem can be studied. For instance, capacity that is installed on edges in the network can also be undirected, i.e. each unit of capacity installed on an edge $\{i, j\}$ has to be shared by traffic on both corresponding $\operatorname{arcs}(i, j)$ and $(j, i)$. A formulation similar to (1)-(5) can be derived in this case. Basically, the constraints (3) and (4) are replaced by a single capacity constraint, summing up all the flows in forward and backward direction (cf. [15] for a flow and path formulation for these cases). Both the directed and the undirected version of the problem are $\mathcal{N} \mathcal{P}$-hard (see Appendix A).

\subsection{Dimension and trivial facets}

With $\operatorname{conv}(D N F M)$ we define the convex hull of all integer solutions of (2)-(5). For this network loading polytope, the dimension and its trivial facets can be specified.

Proposition 1. The dimension of conv (DNFM) is equal to $|E|+|Q|(|A|-|V|+1)$.

Proposition 2. Let $q \in Q$ and $(i, j) \in A$. The trivial inequalities $f_{i j}^{q} \geq 0$ are valid and facet defining for conv $(D N F M)$.

The same results hold for the undirected flow model, whereas similar results can be derived for the path formulations of both variants (see [15] for these results and for proofs of the above stated results). 


\section{Two edge capacity polytopes}

In this paper, we focus on the polyhedral structure of polytopes associated with $\operatorname{conv}(D N F M)$. More precisely, we study the convex hull of sets related to individual edge capacity constraints in the formulations. Whether a flow or path model is considered, whether directed or undirected capacity can be installed, inequalities like (3) and (4) turn up in the formulation. Depending on the variant, for each edge one or two of these inequalities are part of the model. This gives rise to two edge capacity polytopes with associated sets $X$ and $Y$ defined by respectively a single edge capacity constraint and a pair of edge capacity constraints. For DNFM these sets are defined by

$$
\begin{aligned}
X_{i j}^{D F} & =\left\{(x, f) \in \mathbb{Z}_{0}^{+} \times\{0,1\}^{|Q|}: \lambda x_{i j} \geq \sum_{q \in Q} d^{q} f_{i j}^{q}\right\} \\
Y_{i j}^{D F} & =\left\{(x, f) \in \mathbb{Z}_{0}^{+} \times\{0,1\}^{2|Q|}: \lambda x_{i j} \geq \sum_{q \in Q} d^{q} f_{i j}^{q}, \lambda x_{i j} \geq \sum_{q \in Q} d^{q} f_{j i}^{q}\right\} .
\end{aligned}
$$

For the path formulation as well as for other model variants, similar sets can be derived (cf. [15]).

Obviously any valid inequality for these polytopes is valid for the original problem $D N F M$. Moreover, valid inequalities that define a facet of $\operatorname{conv}\left(Y_{i j}^{D F}\right)$, also define a facet of $\operatorname{conv}(D N F M)$.

Theorem 1. Any non-trivial facet defining inequality for $\operatorname{conv}\left(Y_{i j}^{D F}\right)$ is a non-trivial facet defining inequality for conv (DNFM).

Proof. See [15].

For the polytopes $\operatorname{conv}\left(X_{i j}^{D F}\right)$ and $\operatorname{conv}(D N F M)$, such a strong relation is subject to restrictions, formalized in Proposition 3. Here, $Q_{i j}$ denotes the subset of commodities that do not have source $j$ or sink $i$, i.e., $Q_{i j}=\left\{q \in Q: s^{q} \neq i \wedge t^{q} \neq j\right\}$.

Proposition 3. Let $a x \geq b^{T} f-c$ be a non-trivial facet defining inequality for $\operatorname{conv}\left(X_{i j}^{D F}\right)$. It defines a non-trivial facet defining inequality for $\operatorname{conv}(D N F M)$ if and only if for all $\hat{q} \in Q_{j i}$ there exists a subset $\bar{Q} \subseteq Q_{i j} \backslash\{\hat{q}\}$ that satisfies $\sum_{q \in \bar{Q}} b^{q}-c=a \max \left\{\left[d^{\hat{q}}\right\rceil,\left[\sum_{q \in \bar{Q}} d^{q}\right\rceil\right\}$.

Proof. See [15].

Similar results can be derived for the undirected capacity model as well as for the path formulation. Note that, since the dimension of the flow and path formulations differ, these results do not directly follow from the above stated ones.

The edge capacity sets $X_{i j}^{D F}$ and $Y_{i j}^{D F}$ are stated as two examples of edge models occurring in various network loading problems. Although the interpretation of the variables differs on the problem variant and the selected formulation alternative, all these models can be abstracted to two models $X$ and $Y$ defined by respectively a single edge capacity constraint and a pair of edge capacity constraints. Consider a set $Q$ of 
items (commodities) and let $d^{q} \in \mathbb{Q}^{+}$represent the size (demand) for an item $q \in Q$ (normalized to the base capacity $\lambda$ ). Let the integer variable $x$ denote the number of capacity units selected and let the binary variables $f^{q}$ indicate whether or not an individual item $q$ is selected. The undirected edge capacity set $X$ is then defined as

$$
X=\left\{(x, f) \in \mathbb{Z}_{0}^{+} \times\{0,1\}^{|Q|}: x \geq \sum_{q \in Q} d^{q} f^{q}\right\} .
$$

This set is named undirected since it represents the undirected network loading problem restricted to a single edge.

For the directed edge capacity set $Y$, the undirected binary variables $f^{q}$ are replaced by two sets of variables $f^{q}$ and $h^{q}$ for respectively the "forward" and "backward" direction:

$$
Y=\left\{(x, f, h) \in \mathbb{Z}_{0}^{+} \times\{0,1\}^{2|Q|}: x \geq \sum_{q \in Q} d^{q} f^{q}, x \geq \sum_{q \in Q} d^{q} h^{q}\right\} .
$$

The sequel of this paper is devoted to a polyhedral study of the polytopes defined by $X$ and $Y$, in respectively Sect. 4 and Sect. 5 .

We use the following additional notation. Let $D^{q}=\left\lceil d^{q}\right\rceil$ be the smallest integer greater than or equal to $d^{q}$, and let $r^{q}=d^{q}+1-D^{q} \in(0,1]$ be the 'fractional' part of the demand. Similarly, for a subset $S \subseteq Q$, let $d(S)=\sum_{q \in S} d^{q}, D(S)=\lceil d(S)\rceil$ and $r(S)=d(S)+1-D(S)$. Finally, define $e^{S} \in\{0,1\}^{Q}$ as the characteristic vector of a set $S$, i.e. $e_{i}^{S}=1$ if $i \in S$ and zero otherwise. Note that, for each $S \subseteq Q,\left(D(S), e^{S}\right) \in X$. Similarly, for each $S_{1} \subseteq Q, S_{2} \subseteq Q,\left(\max \left\{D\left(S_{1}\right), D\left(S_{2}\right)\right\}, e^{S_{1}}, e^{S_{2}}\right) \in Y$.

\section{The undirected edge capacity polytope}

\subsection{Preliminaries \& characteristics}

The undirected edge capacity polytope has previously been studied by Brockmüller et al. [8] in the context of telecommunication network design, and very recently by Atamtürk and Rajan [1]. The authors of the latter paper obtained to some extend the same (or equivalent) results as we. To avoid overlap, these results are only briefly mentioned in this paper. Proofs can be found in [15].

Given an arbitrary objective function $(\delta, \gamma) \in \mathbb{Z} \times \mathbb{Z}^{|Q|}$, the problem of minimizing the objective over the set $X$ is $\mathcal{N} \mathcal{P}$-hard in general. The polytope $\operatorname{conv}(X)$ is full dimensional and the inequalities $f^{q} \geq 0$ and $f^{q} \leq 1$ define the so-called trivial facets. Brockmüller et al. [8] proved that each non-trivial facet $\operatorname{conv}(X)$ can be written in the form $a x \geq \sum_{q \in Q} b^{q} f^{q}-c$, with $a, c \in \mathbb{Z}_{0}^{+}, b^{q} \in \mathbb{Z}_{0}^{+}$, for all $q \in Q$. A further characterization of the facets is given by the so-called shifting theorem which shows that the complete set of facet defining inequalities for the edge capacity polytope can be obtained from a related edge capacity polytope in which the demands satisfy $d^{q} \in(0,1]$ for all $q \in Q$. The same result was indirectly proven by Atamtürk and Rajan [1].

Theorem 2. (Shifting theorem) Let $i \in Q$ and $\mu \in \mathbb{Z}$ such that $d^{i}+\mu \geq 0$, and define

$$
X(i, \mu)=\left\{(x, f) \in \mathbb{Z}_{0}^{+} \times\{0,1\}^{|Q|}: x \geq\left(d^{i}+\mu\right) f^{i}+\sum_{q \in Q \backslash\{i\}} d^{q} f^{q}\right\} .
$$


Then the inequality

$$
x \geq \sum_{q \in Q} b^{q} f^{q}-c
$$

is a non-trivial facet defining inequality for conv(X) if and only if

$$
x \geq\left(b^{i}+\mu\right) f^{i}+\sum_{q \in Q \backslash\{i\}} b^{q} f^{q}-c
$$

is a non-trivial facet defining inequality for $\operatorname{conv}(X(i, \mu))$.

Proof. Note that we only need to prove that any facet defining inequality for $\operatorname{conv}(X)$ can be converted as indicated to a facet defining inequality for $\operatorname{con} v(X(i, \mu))$, since the converse then directly follows for a suitable choice $\mu^{\prime}=-\mu$. First, we prove validity. Let $(\bar{x}, \bar{f}) \in X(i, \mu)$, then $\left(\bar{x}-\mu \bar{f}^{i}, \bar{f}\right) \in X$, hence $\bar{x}-\mu \bar{f}^{i} \geq \sum_{q \in Q} b^{q} \bar{f}^{q}-c$ which implies that $\bar{x} \geq\left(b^{i}+\mu\right) \bar{f}^{i}+\sum_{q \in Q \backslash\{i\}} b^{q} \bar{f}^{q}-c$. Next, let $\left(x_{1}, f_{1}\right), \ldots,\left(x_{|Q|+1}, f_{|Q|+1}\right)$ be $|Q|+1$ affinely independent solutions of $X$ that satisfy (7) at equality. Then $\left(x_{1}+\right.$ $\left.\mu f_{1}^{i}, f_{1}\right), \ldots,\left(x_{|Q|+1}+\mu f_{|Q|+1}^{i}, f_{|Q|+1}\right)$ satisfy (8) at equality, and they are also affinely independent.

The general characterization of Brockmüller et al. [8] combined with the shifting theorem leads to a range of integer values for the coefficients of the $f^{q}$ variables in any non-trivial facet-defining inequality.

Theorem 3. Let $a x \geq \sum_{q \in Q} b^{q} f^{q}-c$ be a non-trivial facet defining valid inequality for conv $(X)$ with $a, c \in \mathbb{Z}_{0}^{+}$, and $b^{q} \in \mathbb{Z}_{0}^{+}$for all $q \in Q$. Then $b^{q}=a d^{q}$ if $d^{q}$ is integer, and $b^{q} \in\left\{a\left(D^{q}-1\right), \ldots, a D^{q}\right\}$ if $d^{q}$ is not integer, for all $q \in Q$.

Proof. Let $\bar{q} \in Q$ and let $\left(x_{0}, f_{0}\right) \in X$ be a solution with $f_{0}^{\bar{q}}=0$ that satisfies the facet defining inequality at equality. Moreover, let $Q_{0}=\left\{q \in Q: f_{0}^{q}=1\right\}$. Then $a D\left(Q_{0}\right)=\sum_{q \in Q_{0}} b^{q}-c$ and since $\bar{q} \notin Q_{0}$ it follows from validity that $a D\left(Q_{0} \cup\{\bar{q}\}\right) \geq$ $\left(\sum_{q \in Q_{0}} b^{q}-c\right)+b^{\bar{q}}=a D\left(Q_{0}\right)+b^{\bar{q}}$. This yields an upper bound on $b^{\bar{q}}$ since we conclude $b^{\bar{q}} \leq a D\left(Q_{0} \cup\{\bar{q}\}\right)-a D\left(Q_{0}\right)$.

Similarly, let $\left(x_{1}, f_{1}\right) \in X$ be a solution with $f_{1}^{\bar{q}}=1$ that satisfies the facet defining inequality at equality, and define $Q_{1}=\left\{q \in Q: f_{1}^{q}=1\right\}$. Again, $a D\left(Q_{1}\right)=$ $\sum_{q \in Q_{1}} b^{q}-c$ and since $\bar{q} \in Q_{1}$ it follows from validity that $a D\left(Q_{1} \backslash\{\bar{q}\}\right) \geq$ $\left(\sum_{q \in Q_{1}} b^{q}-c\right)-b^{\bar{q}}=a D\left(Q_{1}\right)-b^{\bar{q}}$. This implies a lower bound on $b^{\bar{q}}$, namely $b^{\bar{q}} \geq a D\left(Q_{1}\right)-a D\left(Q_{1} \backslash\{\bar{q}\}\right)$.

If $d^{\bar{q}}$ is integer, then both the lower and upper bound are equal to $a d^{\bar{q}}$ which proves the first part of our claim. If $d^{\bar{q}}$ is not integer, then $b^{\bar{q}} \leq a D\left(Q_{0} \cup\{\bar{q}\}\right)-a D\left(Q_{0}\right) \leq a D^{\bar{q}}$ and $b^{\bar{q}} \geq a D\left(Q_{1}\right)-a D\left(Q_{1} \backslash\{\bar{q}\}\right) \geq a\left(D^{\bar{q}}-1\right)$.

From Theorem 3, we can derive necessary and sufficient conditions under which the inequalities $x \geq \sum_{q \in Q} d^{q} f^{q}$ (called the model inequality) and $0 \leq f^{q} \leq 1$ for all $q \in Q$ yield the complete description of the edge capacity polytope $X$. 
Theorem 4. The model inequality is the unique non-trivial facet defining inequality for the polyhedron conv $(X)$ if and only if $d^{q} \in \mathbb{Z}_{0}^{+}$, for all $q \in Q$.

Proof. If $d^{\hat{q}} \notin \mathbb{Z}_{0}^{+}$, for some $\hat{q} \in Q$, then the fractional solution $(x, f)=\left(d^{q}, e^{\{\hat{q}\}}\right)$ is an extreme point of the LP-relaxation that satisfies all the model inequalities. If $d^{q} \in \mathbb{Z}_{0}^{+}$ for all $q \in Q$, then by Theorem 3 we have that in every inequality $x \geq \sum_{q \in Q} b^{q} f^{q}-c$ that defines a non-trivial facet of $\operatorname{conv}(X), b^{q}=d^{q}$ for all $q \in Q$. If $c>0$, then the resulting inequality is dominated by the model inequality. Hence, the model inequality $x \geq \sum_{q \in Q} d^{q} f^{q}$ defines the only non-trivial facet in this case.

The main consequence of Theorem 2, however, is that we can restrict ourselves in the sequel to $d^{q} \in(0,1]$. Afterwards, each inequality can simply be shifted back.

In Brockmüller et al. [8], the class of $c$-strong inequalities is introduced. A set $S \subseteq Q$ is called c-strong if $c=\sum_{q \in S} D^{q}-D(S)$. The set is maximal $c$-strong if $S \backslash\{i\}$ is $c$-strong for all $i \in S$, and $S \cup\{i\}$ is not $c$-strong for all $i \in Q \backslash S$. Given a $c$-strong set $S$, the $c$-strong inequality is defined as

$$
x \geq \sum_{q \in S} D^{q} f^{q}+\sum_{q \in Q \backslash S}\left(D^{q}-1\right) f^{q}-c .
$$

Inequality (9) is valid for all $S$ and defines a facet of $\operatorname{conv}(X)$ if and only if $S$ is maximal $c$-strong. By Theorem 3, the class of $c$-strong inequalities covers all inequalities with $a=1$ in the general form with only integer coefficients.

In Atamtürk and Rajan [1], the $c$-strong inequalities are generalized to the class of $k$-split $c$-strong inequalities. In the next subsection, the class of lower convex envelope inequalities is derived, whereas Sect. 4.3 is devoted to lifted knapsack cover inequalities. The latter class was also derived in [1]. Both classes include the class of $c$-strong inequalities.

\subsection{Lower convex envelope inequalities}

A lower convex envelope inequality is defined on a projection of the set $X$. We show two different types of facet defining inequalities that may arise in the class of lower convex envelope inequalities. Moreover, we show that lifting lower convex envelope inequalities to obtain valid inequalities for $X$ itself can be performed in polynomial time. We start with the definition of a projection of the edge capacity polytope.

Definition 1. Let $Q^{0}, Q^{1} \subset Q$ be disjoint subsets of $Q$. Then $X\left(Q^{0}, Q^{1}\right)$ defined by

$$
X\left(Q^{0}, Q^{1}\right)=\left\{(x, f) \in X: f^{q}=0 \forall q \in Q^{0}, f^{q}=1 \forall q \in Q^{1}\right\}
$$

is the projection of $X$ on the space with $f^{q}=0$ for all $q \in Q^{0}$ and $f^{q}=1$ for all $q \in Q^{1}$. 
This projected edge set can be seen as a set of vectors in $(|Q|+1)$-dimensional space. Instead of representing the set $X\left(Q^{0}, Q^{1}\right)$ in $(|Q|+1)$-dimensional space, one can also plot all vectors in $X\left(Q^{0}, Q^{1}\right)$ in two-dimensional space, as in the example of Fig. 1. Let $S=Q \backslash\left(Q^{0} \cup Q^{1}\right)$. The horizontal axis of this figure measures $\sum_{q \in S} f^{q}$ and the vertical axis measures the value of the capacity variable $x$. Hence, a solution $(x, f) \in X\left(Q^{0}, Q^{1}\right)$ is represented by a point with coordinates $\left(\sum_{q \in S} f^{q}, x\right)$ in the two-dimensional figure. Similarly as in $(|Q|+1)$-dimensional space, an inequality $a x \geq b \sum_{q \in S} f^{q}-c$ that is satisfied by all solutions in this two-dimensional space yields a valid inequality for the set $X\left(Q^{0}, Q^{1}\right)$. Note that these inequalities have the same coefficient for all $q \in S$ in the inequality. We consider the strongest possible (non-redundant) valid inequalities arising from the two-dimensional space. These inequalities describe the lower convex envelope of the set of solutions in $X\left(Q^{0}, Q^{1}\right)$, as indicated in Fig. 1.

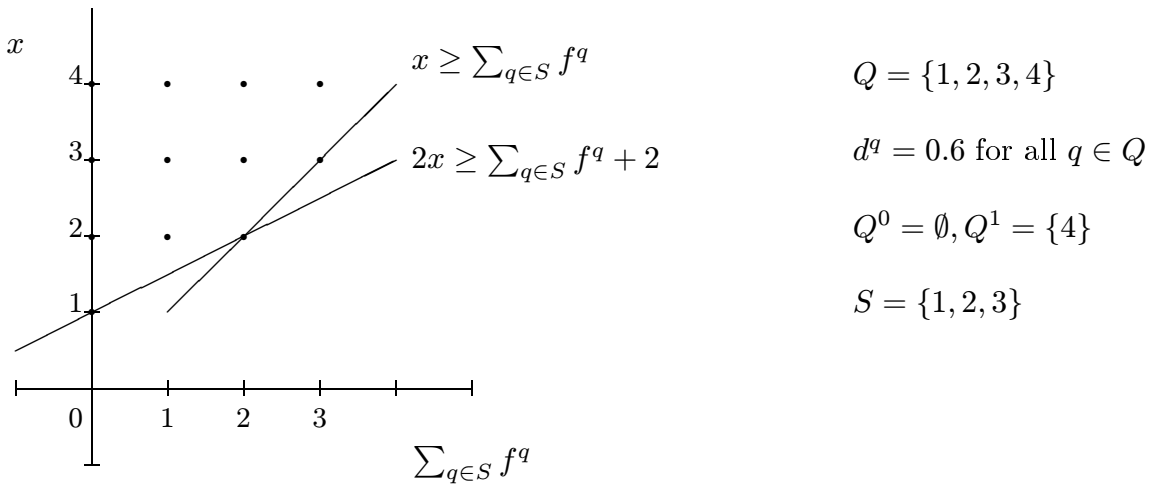

Fig. 1. Lower convex envelope inequalities

Assume that the commodities in $S$ are ordered such that $d^{1} \leq d^{2} \leq \ldots \leq d^{|S|}$, and for $k=1, \ldots,|S|$, let $S_{k}=\{1, \ldots, k\}$. Then the set of lower convex envelope inequalities basically describes the lower convex envelope of the points $\left(k, D\left(Q^{1} \cup S_{k}\right)\right)$ for $k=1, \ldots,|S|$ in two-dimensional space. The following proposition states bounds on the slope of a lower convex envelope inequality.

Proposition 4. Let $a x \geq b \sum_{q \in S} f^{q}-c$ be a non-dominated lower convex envelope inequality for the set $X\left(Q^{0}, Q^{1}\right)$ with demand values $d^{q}=r^{q} \in(0,1]$ for all $q \in Q$. Then the slope $b / a$ of the lower convex envelope inequality satisfies $0 \leq b / a \leq 1$. Moreover, $a, b$, and c can be selected in such a way that $a, b, c \in \mathbb{Z}_{0}^{+}$and $a \leq|S|$.

Proof. Each non-dominated lower convex envelope inequality is defined by two points, say $\left(k_{1}, D\left(Q^{1} \cup S_{k_{1}}\right)\right)$ and $\left(k_{2}, D\left(Q^{1} \cup S_{k_{2}}\right)\right)$ in the two-dimensional figure, for some $k_{1}, k_{2} \in\{0, \ldots,|S|\}$, with $k_{1}<k_{2}$. The slope $b / a$ of such a line is then the quotient of $D\left(Q^{1} \cup S_{k_{2}}\right)-D\left(Q^{1} \cup S_{k_{1}}\right)$ and $k_{2}-k_{1}$. Since $S_{k_{1}} \subseteq S_{k_{2}}$ it follows that the numerator is nonnegative, which together with $k_{1} \leq k_{2}$ implies that $b / a \geq 0$. Furthermore, $S_{k_{1}} \subseteq S_{k_{2}}$ together with $d^{q} \in(0,1]$ for all $q \in S$ implies that $D\left(Q^{1} \cup S_{k_{2}}\right)-D\left(Q^{1} \cup S_{k_{1}}\right) \leq k_{2}-k_{1}$. 
Hence, $b / a \leq 1$. Finally, both the numerator and denominator are bounded by $|S|$. Hence, the inequality can be written in a form with $a, b, c \in \mathbb{Z}_{0}^{+}$and $a \leq|S|$.

Next, we derive necessary and sufficient conditions under which there exists a lower convex envelope inequality $a x \geq b \sum_{q \in S} f^{q}-c$ with slope $b / a=1$ that is also facet defining for $\operatorname{conv}\left(X\left(Q^{0}, Q^{1}\right)\right)$. Let $n\left(Q^{1}, S\right):=\sum_{q \in S} D^{q}+D\left(Q^{1}\right)-D\left(S \cup Q^{1}\right)$ with $Q^{1} \subset Q$ and $S \subset Q \backslash Q^{1}$. In words, $n\left(Q^{1}, S\right)$ measures the surplus in capacity for the set of items $Q^{1} \cup S$ if $D\left(Q^{1}\right)$ units of capacity have already been 'installed' for the set of items $Q^{1}$ and an additional $D^{q}$ units of capacity are installed for each item $q \in S$. Note that the value of $n\left(Q^{1}, S\right)$ does not change if demand data $r^{q} \in(0,1]$ are considered instead of the real demand data $d^{q}$, for all $q \in Q$.

Since $b / a \leq 1$, it is always possible, by an appropriate choice of $c$, to construct a valid lower convex envelope inequality with $b / a=1$. The strongest lower convex envelope is obtained by selecting $c$ in such a way that the inequality is adjacent to the point $\left(|S|, D\left(Q^{1} \cup S\right)\right)$. Theorem 5 states which other condition should be satisfied by the inequality to be facet defining. In words this condition states that for every $q \in S$, the capacity needed for $Q^{1} \cup S$ is one larger than the capacity needed for $Q^{1} \cup S \backslash\{q\}$. Note that, since the lower convex envelope inequalities with $b / a=1$ are in fact $c$-strong inequalities, this condition is equivalent to the condition for $c$-strong inequalities to be facet defining.

Theorem 5. Let $Q^{0}, Q^{1}, S$ be a partition of $Q, d^{q} \in(0,1]$ for all $q \in Q$, and let $a x \geq b \sum_{q \in S} f^{q}-c$ be the strongest lower convex envelope inequality with slope $b / a=1$ for the edge capacity set $X\left(Q^{0}, Q^{1}\right)$. Then $c=n\left(Q^{1}, S\right)-D\left(Q^{1}\right)$, resulting in the inequality.

$$
x \geq \sum_{q \in S} f^{q}+D\left(Q^{1}\right)-n\left(Q^{1}, S\right)
$$

It defines a facet of $\operatorname{conv}\left(X\left(Q^{0}, Q^{1}\right)\right)$ if and only if $D\left(Q^{1} \cup S \backslash\{q\}\right)=D\left(Q^{1} \cup S\right)-1$ for all $q \in S$.

Proof. The value of $c$ directly follows from the fact that $\left(D\left(Q^{1} \cup S\right), e^{S}\right)$ satisfies (10) with equality.

Next, we prove that the remaining conditions are sufficient to guarantee that the inequality is facet defining for $\operatorname{conv}\left(X\left(Q^{0}, Q^{1}\right)\right)$, which has dimension $|S|+1$. This follows from the fact that the vectors $\left(D\left(Q^{1} \cup S\right), e^{Q^{1} \cup S}\right)$ and $\left(D\left(Q^{1} \cup S \backslash\{q\}\right), e^{Q^{1} \cup S \backslash\{q\}}\right)$ for all $q \in S$ yield $|S|+1$ affinely independent vectors in $X\left(Q^{0}, Q^{1}\right)$ that satisfy that inequality at equality.

Conversely, let $\hat{q} \in S$. If $D\left(Q^{1} \cup S \backslash\{\hat{q}\}\right) \neq D\left(Q^{1} \cup S\right)-1$ then the solution $(x, f)=\left(D\left(Q^{1} \cup S \backslash\{\hat{q}\}\right), e^{Q^{1} \cup S \backslash\{\hat{q}\}}\right)$ is not on the face of the valid inequality $x \geq$ $\sum_{q \in S} f^{q}+D\left(Q^{1}\right)-n\left(Q^{1}, S\right)$. For any set $T \subseteq S \backslash\{\hat{q}\}$ it holds that $D\left(Q^{1} \cup S \backslash\{\hat{q}\}\right) \leq$ $D\left(Q^{1} \cup T\right)+|S \backslash(T \cup\{\hat{q}\})|$. Hence, the vector $\left(D\left(Q^{1} \cup T\right), e^{Q^{1} \cup T}\right)$ does not satisfy the inequality at equality, since $D\left(Q^{1} \cup T\right) \geq D\left(Q^{1} \cup S \backslash\{\hat{q}\}\right)-|S \backslash(T \cup\{\hat{q}\})|>$ $|S \backslash\{\hat{q}\}|-c-|S \backslash(T \cup\{\hat{q}\})|=|T|-c$. Thus there exists no solution $(x, f) \in X\left(Q^{0}, Q^{1}\right)$ with $f^{\hat{q}}=0$ that satisfies the inequality at equality. As a result, the face of the inequality 
is a subset of the face defined by the inequality $f^{\hat{q}} \leq 1$, which implies that the inequality is not facet defining.

Figure 1 shows a facet defining lower convex envelope inequality with slope $b / a=1$. Also the "last but one" lower convex envelope inequality can define a facet of $\operatorname{conv}\left(X\left(Q^{0}, Q^{1}\right)\right)$.

Theorem 6. Consider $X\left(Q^{0}, Q^{1}\right)$ for demand data $d^{q}=r^{q} \in(0,1]$. Assume that $D\left(Q^{1} \cup S \backslash\{q\}\right)=D\left(Q^{1} \cup S\right)-1$ for all $q \in S$. If $D\left(Q^{1} \cup S_{|S|-1}\right)=D\left(Q^{1} \cup S_{|S|-2}\right)$, then the inequality

$$
\alpha x \geq \sum_{q \in S} f^{q}+\alpha D\left(Q^{1} \cup S\right)-(|S|+\alpha-1)
$$

with

$$
1 / \alpha=\max _{k=0, \ldots,|S|-2}\left\{\frac{D\left(Q^{1} \cup S\right)-1-D\left(Q^{1} \cup S_{k}\right)}{|S|-1-k}\right\}
$$

defines a second lower convex envelope inequality through the point $\left(|S|-1, D\left(Q^{1} \cup\right.\right.$ $\left.\left.S_{|S|-1}\right)\right)$. Moreover, it defines a facet of conv $\left(X\left(Q^{0}, Q^{1}\right)\right)$.

Proof. It is easy to verify that the inequality (11) goes through the point $(|S|-1$, $\left.D\left(Q^{1} \cup S\right)-1\right)$, independently of the value $\alpha$. Since $D\left(Q^{1} \cup S_{|S|-1}\right)=D\left(Q^{1} \cup S_{|S|-2}\right)$, the point $\left(|S|-2, D\left(Q^{1} \cup S_{|S|-2}\right)\right)$ is not on the same line as $\left(|S|, D\left(Q^{1} \cup S\right)\right)$ and $\left(|S|-1, D\left(Q^{1} \cup S\right)-1\right)$. Hence, values $\alpha>1$ exist such that (11) is a valid lower convex envelope inequality, different from (10). The strongest inequality (11) with $\alpha>1$ goes through a second point $\left(k, D\left(Q^{1} \cup S_{k}\right)\right)$ for some $k \in\{0, \ldots,|S|-2\}$. Since the lower convex envelope inequality must be valid, it follows that its slope is equal to the maximal slope between point $\left(|S|-1, D\left(Q^{1} \cup S\right)-1\right)$ and $\left(k, D\left(Q^{1} \cup S_{k}\right)\right)$, over all $k \in\{0, \ldots,|S|-2\}$.

The fact that (11) is facet defining for $\operatorname{conv}\left(X\left(Q^{0}, Q^{1}\right)\right)$ follows from the fact that the solution vectors $\left(D\left(Q^{1} \cup S \backslash\{q\}\right), e^{Q^{1} \cup S \backslash\{q\}}\right)$ for all $q \in S$ all satisfy the inequality at equality. Moreover, for the $k$ that maximizes $\alpha$, the vector $(x, f)=\left(D\left(Q^{1} \cup S_{k}\right), e^{Q^{1} \cup S_{k}}\right)$ also satisfies the inequality at equality. This yields $|S|+1$ affinely independent vectors in $X\left(Q^{0}, Q^{1}\right)$ on the face of the inequality, hence it defines a facet.

Lower convex envelope inequalities for $X\left(Q^{0}, Q^{1}\right)$ have to be lifted to $X$. Theorem 7 states that this can be done polynomial time.

Theorem 7. Let $a x \geq b \sum_{q \in S} f^{q}-c$ be a lower convex envelope inequality for $\operatorname{conv}\left(X\left(Q^{0}, Q^{1}\right)\right)$ with demand data $d^{q}=r^{q} \in(0,1]$ for all $q \in S$. Given a sequence of the commodities in $Q^{0} \cup Q^{1}$, maximal sequential lifting of this inequality to obtain a valid inequality for conv $(X)$ can be done in polynomial time. 
Proof. By Proposition 4, we may assume without loss of generality that $a, b, c \in \mathbb{Z}_{0}^{+}$ with $b \leq a \leq|S|$. By Theorem 3, the lifting coefficients $b^{q}$ obtained by maximal sequential lifting of variables in $q \in Q^{0} \cup Q^{1}$ also satisfy $b^{q} \leq a \leq|S|$.

Now consider the lifting process for a variable $f^{\hat{q}}, \hat{q} \in \bar{Q}^{0}$, then the value of the lifting coefficient $b^{\hat{q}}$ is given by

$$
b^{\hat{q}}=\min _{(x, f) \in X\left(Q^{0} \backslash\{\hat{q}\}, Q^{1}\right): f{ }^{\hat{q}}=1}\left\{a x-b \sum_{q \in S} f^{q}+c\right\} .
$$

For a fixed value of $x$, the problem simplifies to a knapsack problem. Since $d^{q} \in(0,1]$, $x$ is bounded from above by $|Q|$. Hence, the problem can be solved by solving at most $|Q|$ knapsack problems. Even stronger, by using dynamic programming techniques, all $|Q|$ knapsack problems can be solved by solving the one with largest $x$. Since the optimal value is bounded by $|S| \leq|Q|$, this knapsack problem can be solved in $\mathcal{O}\left(|Q|^{2}\right)$. Hence, the lifting coefficient can thus be determined in $\mathcal{O}\left(|Q|^{2}\right)$ time. A similar argument holds for the lifting of a variable in $Q^{1}$. Since the total number of variables to be lifted is bounded by $|Q|$, the complete lifting process can be performed in $\mathcal{O}\left(|Q|^{3}\right)$ time.

\subsection{Integer lifting of knapsack inequalities}

A different approach to obtain the two special cases of valid lower convex envelope inequalities (10) and (11) is stated in this subsection. Using a result of Wolsey [25], valid inequalities for the edge capacity polytope can be obtained from valid inequalities for a related 0-1 knapsack polytope by integer lifting techniques.

Definition 2. Let $Q^{0}, Q^{1}, S$ be a partition of $Q$, and let $\bar{x} \in \mathbb{Z}_{0}^{+}$with $\bar{x} \geq D\left(Q^{1} \cup\{q\}\right)$ for all $q \in S$. Let $b=\bar{x}-d\left(Q^{1}\right)$, then $X\left(Q^{0}, Q^{1}, \bar{x}\right)$ is a knapsack set defined by

$$
\begin{aligned}
X\left(Q^{0}, Q^{1}, \bar{x}\right) & =\left\{(x, f) \in X: f^{q}=0 \forall q \in Q^{0}, f^{q}=1 \forall q \in Q^{1}, x=\bar{x}\right\} \\
& \simeq\left\{f \in\{0,1\}^{|S|}: \sum_{q \in S} d^{q} f^{q} \leq b\right\} .
\end{aligned}
$$

The set $X\left(Q^{0}, Q^{1}, \bar{x}\right)$ is the projection of $X$ on the space with $f^{q}=0$ for all $q \in Q^{0}$, $f^{q}=1$ for all $q \in Q^{1}$, and $x=\bar{x}$. Note that the condition $\bar{x} \geq D\left(Q^{1} \cup\{q\}\right)$ for all $q \in S$ implies that $\operatorname{dim}\left(\operatorname{conv}\left(X\left(Q^{0}, Q^{1}, \bar{x}\right)\right)\right)=|S|$, that is, the knapsack polytope $X\left(Q^{0}, Q^{1}, \bar{x}\right)$ is full dimensional.

Given a valid inequality for $X\left(Q^{0}, Q^{1}, \bar{x}\right)$, we can lift it to multiple valid inequalities for $X\left(Q^{0}, Q^{1}\right)$ :

Theorem 8. (cf. Wolsey [25]) Let $Q^{0}, Q^{1}$, S be a partition of the set $Q$, and let $\bar{x} \in \mathbb{Z}_{0}^{+}$ be an integer with $\bar{x} \geq D\left(Q^{1} \cup\{q\}\right)$ for all $q \in S$. If $\sum_{q \in S} \pi^{q} f^{q} \leq \pi^{0}$ is a valid inequality for $X\left(Q^{0}, Q^{1}, \bar{x}\right)$ then $\sum_{q \in S} \pi^{q} f^{q} \leq \pi^{0}+\alpha(x-\bar{x})$ is a valid inequality for $X\left(Q^{0}, Q^{1}\right)$ if and only if

$$
\alpha^{L}:=\max _{x \in \mathbb{Z}_{0}^{+}: x>\bar{x}}\left\{\frac{\eta(x)-\pi^{0}}{x-\bar{x}}\right\} \leq \alpha \leq \min _{x \in \mathbb{Z}_{0}^{+}: D\left(Q^{1}\right) \leq x<\bar{x}}\left\{\frac{\pi^{0}-\eta(x)}{\bar{x}-x}\right\}=: \alpha^{U}
$$


where

$$
\eta(x)=\max \left\{\sum_{q \in S} \pi^{q} f^{q}: \sum_{q \in S} d^{q} f^{q} \leq x-d\left(Q^{1}\right), f^{q} \in\{0,1\}, \forall q \in S\right\} .
$$

Moreover, if $\sum_{q \in S} \pi^{q} f^{q} \leq \pi^{0}$ is a facet defining inequality for $\operatorname{conv}\left(X\left(Q^{0}, Q^{1}, \bar{x}\right)\right)$ and $\alpha^{L} \leq \alpha^{U}$, then $\sum_{q \in S} \pi^{q} f^{q} \leq \pi^{0}+\alpha^{L}(x-\bar{x})$ and $\sum_{q \in S} \pi^{q} f^{q} \leq \pi^{0}+\alpha^{U}(x-\bar{x})$ are facet defining inequalities for $\operatorname{conv}\left(X\left(Q^{0}, Q^{1}\right)\right)$.

The problem to be solved to determine $\eta(x)$ is a knapsack problem which can be solved by dynamic programming in pseudo-polynomial time. In fact, the values $\eta(x)$ can be obtained by a single run of the dynamic program for a sufficiently large value of $x$, since all smaller values are calculated during this run. In general, $\alpha^{L} \leq \alpha^{U}$ does not necessarily hold, in which case integer lifting is not possible. For the special case of the well-known cover inequalities [2,21,24] for the knapsack polytope, however, integer lifting is always possible and can be done in polynomial time (provided that $d^{q} \in(0,1]$ for all $q \in Q$ ).

Theorem 9. Let $d^{q} \in(0,1]$ for all $q \in Q$, let $Q^{0}, Q^{1}, S$ be a partition of the set $Q$ such that $S \neq \emptyset$, and let $\bar{x} \in \mathbb{Z}_{0}^{+}$satisfy $\bar{x} \geq D\left(Q^{1} \cup\{q\}\right)$ for all $q \in S$. If $S$ is a minimal cover for the knapsack polytope $X\left(Q^{0}, Q^{1}, \bar{x}\right)$, then

(i) integer lifting of the minimal cover inequality can be done in polynomial time,

(ii) $1=\alpha^{L} \leq \alpha^{U}$,

(iii) $\alpha^{U}>1$ if and only if $D\left(Q^{1} \cup S_{|S|-1}\right)=D\left(Q^{1} \cup S_{|S|-2}\right)$,

(iv) the resulting facet defining inequalities for $\operatorname{conv}\left(X\left(Q^{0}, Q^{1}\right)\right)$ are:

$$
\begin{aligned}
x & \geq \sum_{q \in S} f^{q}+D\left(Q^{1}\right)-n\left(Q^{1}, S\right) \\
\alpha^{U} x & \geq \sum_{q \in S} f^{q}+\alpha^{U} D\left(Q^{1} \cup S\right)-\left(|S|+\alpha^{U}-1\right) .
\end{aligned}
$$

Proof. The knapsack problem that needs to be solved in order to determine the lifting coefficients has the same objective coefficient for all items. Hence, a sorting algorithm can solve the knapsack problem in polynomial time. Since $S$ is a minimal cover, $\sum_{q \in S \backslash\{i\}} d^{q} \leq \bar{x}-d\left(Q^{1}\right)$ for all $i \in S$. Hence, by $d^{q} \in(0,1]$ for all $q \in Q$ it follows that $\eta(x)=|S|$ for all $x>\bar{x}$, and hence, the maximum value for $\alpha^{L}$ is attained for $x=\bar{x}+1$, which yields $\alpha^{L}=1$. For $x<\bar{x}$, it is easy to see that $\eta(x) \leq \eta(x+1)-1$. Therefore, for $x<\bar{x}$ it holds that $\left(\pi^{0}-\eta(x)\right) /(\bar{x}-x) \geq(\bar{x}-x) /(\bar{x}-x)=1$, such that $\alpha^{U} \geq \alpha^{L}$.

Next, if $D\left(Q^{1} \cup S_{|S|-1}\right)>D\left(Q^{1} \cup S_{|S|-2}\right)$ then there exist $i, j \in S$ such that $n\left(Q^{1}, S \backslash\{i, j\}\right)=n\left(Q^{1}, S \backslash\{i\}\right)$, which implies $\eta(\bar{x}-1)=|S|-2$, and hence $\alpha^{U} \leq 1$. Together with $\alpha^{U} \geq \alpha^{L}=1$ this yields $\alpha^{U}=1$. Conversely, if $n\left(Q^{1}, S \backslash\{i, j\}\right) \neq$ $n\left(Q^{1}, S \backslash\{i\}\right)$ for all $i, j \in S$, then $\eta(\bar{x}-1) \leq|S|-3$. Hence, for $x=\bar{x}-1$ the quotient $\left(\pi^{0}-\eta(x)\right) /(\bar{x}-x)$ is strictly greater than 1 . Moreover, again using $\eta(x) \leq \eta(x+1)-1$ it follows that the quotient can never attain the value 1 , for $x<\bar{x}$.

Finally, since $S$ is a minimal cover and $d^{q} \in(0,1]$ for all $q \in S$, it follows that $\bar{x}=D\left(Q^{1} \cup S\right)-1$. Substitution of this value in the inequalities of Theorem 8 yields the required inequalities. 
Note that (12) and (13) are equivalent to (10) and (11), respectively. As a consequence, Theorem 7 can be applied implying that maximal lifting of (12) and (13) can be done in polynomial time as well. This in contrast to the lifting problem of minimal covers for the knapsack polytope, which is still open (cf. [12]).

Moreover, note that Theorem 9 does not hold for general demands $d^{q}$. In [15], an example is presented where shifting to $(0,1]$ and back results in two facet defining valid inequalities, whereas direct application of the integer lifting technique on the cover inequality gives $\alpha^{U}<\alpha^{L}$, and hence, no valid inequalities can be found.

\section{The directed edge capacity polytope}

In this section, we derive a class of valid inequalities for the directed edge capacity polytope $\operatorname{conv}(Y)$ and identify conditions under which the valid inequalities are facet defining. Next, we show that sequential lifting for the directed edge capacity polytope is $\mathcal{N} \mathcal{P}$-hard in general, even for lifting orders which first lift all flow variables in the same direction.

Proposition 5. Let $\hat{q} \in Q$ and let $\alpha \in \mathbb{Z}_{0}^{+}$such that $1 \leq \alpha \leq D^{\hat{q}}$. Then

$$
x \geq \alpha f^{\hat{q}}+\sum_{q \in Q}\left(D^{q}-\alpha\right) h^{q}
$$

is a valid inequality for $Y$.

Proof. Consider an arbitrary feasible solution $(\bar{x}, \bar{f}, \bar{h}) \in Y$ and let $\bar{Q}=\left\{q \in Q: \bar{h}^{q}=1\right\}$. If $|\bar{Q}|=0$, then $\bar{x} \geq D^{\hat{q}} \bar{f}^{\hat{q}} \geq \alpha \bar{f}^{\hat{q}}=\alpha \bar{f}^{\hat{q}}+\sum_{q \in Q}\left(D^{q}-\alpha\right) \bar{h}^{q}$. If $|\bar{Q}| \geq 1$, then

$$
\begin{aligned}
\bar{x} & \geq\left\lceil\max \left\{\sum_{q \in Q} d^{q} \bar{f}^{q}, \sum_{q \in Q} d^{q} \bar{h}^{q}\right\}\right\rceil \geq D(\bar{Q}) \\
& =\sum_{q \in \bar{Q}}\left(D^{q}-\alpha\right)+\sum_{q \in \bar{Q}}(\alpha-1)+\left\lceil\sum_{q \in \bar{Q}} r^{q}\right\rceil \\
& \geq \sum_{q \in \bar{Q}}\left(D^{q}-\alpha\right)+(\alpha-1)+1 \geq \alpha \bar{f}^{\hat{q}}+\sum_{q \in Q}\left(D^{q}-\alpha\right) \bar{h}^{q}
\end{aligned}
$$

which proves our claim.

Next, in Proposition 6, we derive a sufficient condition for the inequality (14) to be facet defining.

Proposition 6. Let $\hat{q} \in Q$. If $\alpha=1, D^{q} \geq D^{\hat{q}}$ for all $q \in Q$, and for all $q \in Q \backslash\{\hat{q}\}$ there exists a subset $\tilde{Q} \subseteq Q$, with $|\tilde{Q}|=2,\left\lceil\sum_{q \in \tilde{Q}} r^{q}\right\rceil=1$, and $D(\tilde{Q}) \geq D(\{q, \hat{q}\})$, then (14) is facet defining for $\operatorname{conv}(Y)$.

Proof. The dimension of $\operatorname{conv}(Y)$ is $2|Q|+1$. We give $2|Q|$ affinely independent vectors in $Y$ satisfying the inequality at equality. These vectors are $(x, f, h)=(0,0,0)$, $(x, f, h)=\left(D^{q}, e^{\hat{q}}, e^{q}\right)$, for all $q \in Q$, and $(x, f, h)=\left(D(\tilde{Q}), e^{\hat{q} \cup q}, e^{\tilde{Q}}\right)$, for all $q \in Q \backslash\{\hat{q}\}$. 
Example 1. Consider an instance of the set $Y$ with $|Q|=4$ and the demands are $d^{1}=1.8, d^{2}=2.1, d^{3}=2.6, d^{4}=3.2$. If $\hat{q}=1$, then the inequalities

$$
\begin{aligned}
& x \geq f^{1}+h^{1}+2 h^{2}+2 h^{3}+3 h^{4} \\
& x \geq h^{1}+f^{1}+2 f^{2}+2 f^{3}+3 f^{4}
\end{aligned}
$$

are valid and facet defining for $\operatorname{conv}(Y)$.

In contrast to the lifting of lower convex envelope inequalities, the lifting of a valid inequality for the directed model under an arbitrary lifting order is an $\mathcal{N} \mathcal{P}$-hard problem in general.

Proposition 7. Lifting of a valid inequality for the directed edge capacity polytope is $\mathcal{N} \mathcal{P}$-complete.

Proof. See Appendix B.

\section{Computational analysis}

To test the effect of the developed theory on the solvability of network loading problems, we implemented a branch-and-cut algorithm, using A Branch-And-CUt System (ABACUS), version 2.2 [22], in combination with CPLEX 6.5 [16]. The algorithm was executed on a Sun Ultra-1 m140 workstation with $128 \mathrm{MB}$ internal memory. The program was tested on two sets of instances. First, we tested the quality of the valid inequalities for the edge capacity polytope with a set of randomly generated edge capacity instances. Second, we compared the results of the Branch-and-Cut algorithm with/without these inequalities for real-life instances of the DNFM model from KPN Research, The Netherlands. We start this section with a discussion of the computational aspects of the separation of the different sets of inequalities.

\subsection{Separation of valid inequalities}

For the undirected edge capacity polytope, the classes of $c$-strong, lower convex envelope, and integer lifted knapsack covers have to be separated, and for the directed edge capacity polytope, the two-side inequalities have to be separated. Moreover, for network loading problems also the well-known classes of cut-set and 3 partition inequalities can be separated.

The separation problem for the already known $c$-strong inequalities is described by Brockmüller et al. [8]. They show that for a given value of $c$, finding the most violated $c$-strong inequality requires solving a knapsack problem (Atamtürk and Rajan [1] formally proved that the separation problem is $\mathcal{N} \mathcal{P}$-hard). They propose heuristic methods to find the most violated inequality for values $c=0,1,2$. The best results were obtained with a greedy algorithm where the commodities are selected in non-decreasing order of $\bar{f}_{i j}^{q} r^{q}$. Our computational experiments support their findings that this method yields good results. 
For any $Q^{0}, Q^{1}$ the lower convex envelope inequalities can be separated by enumeration. However, it is still an open question whether the best $Q^{0}$ and $Q^{1}$ can be found in polynomial time. Moreover, the complexity to determine the order in which the variables of $Q^{0}$ and $Q^{1}$ have to be lifted to obtain the most violated inequality is open as well. For a given LP-solution $(\bar{x}, \bar{f})$ and an arbitrary $\operatorname{arc}(i, j) \in A$, we adopted the following rule for the partition of $Q: S=\left\{q \in Q: 0<\bar{f}_{i j}^{q} \leq 1\right\}, Q^{0}=\left\{q \in Q: \bar{f}_{i j}^{q}=0\right\}$ and $Q^{1}=\emptyset$. In this way, the highest value for $\sum_{q \in S} f^{q}$ is obtained. Moreover, since $\bar{f}_{i j}^{q}=0$ for all $q \in Q^{0}$, the (current) violation of the inequality is not influenced by the lifting order of the projected variables. The variables of $Q^{0}$ are lifted in order of nonincreasing reduced cost. Note that, this separation heuristic selects only lower convex envelope inequalities of the subclass defined by $Q^{1}=\emptyset$.

For the integer lifted knapsack covers, again the questions raise how to partition $Q$ in $Q^{0}, Q^{1}$ and $S$ such that $S$ defines a (minimal) cover, and in which order the variables in $Q^{0}$ and $Q^{1}$ have to be lifted in order to obtain the most violated inequality. For a given LP-solution $(\bar{x}, \bar{f})$, we consider for every arc $(i, j) \in A$ the knapsack polytope $X\left(Q^{0}, Q^{1}, \tilde{x}\right)$, with $Q^{0}=\left\{q \in Q: \bar{f}_{i j}^{q}=0\right\}, Q^{1}=\left\{q \in Q: \bar{f}_{i j}^{q}=1\right\}$, and $\tilde{x}=\lceil\bar{x}\rceil$. The search for a violated cover inequality is done in the way proposed by $\mathrm{Gu}$ et al. [13]. After a violated cover is found, first the $x$ variable is lifted, and afterwards the remaining flow variables (those not in the cover are included in the set $Q^{0}$ ) according to the order defined in [13]: first lift the variables in $Q^{1}$, next those in $Q^{0}$, both in order of non-increasing magnitude of reduced costs.

For the directed edge capacity polytope, the class of two-side inequalities have to be separated. For a given arc $(i, j) \in A$ and a fixed value of $\alpha$, finding the most violated two-side inequality (14) is an easy task. For a given LP-solution $(\bar{x}, \bar{f})$ a violated two-side inequality exists if and only if there exists an element $\hat{q} \in Q$ such that $\alpha \bar{f}_{i j}^{\hat{q}}>\bar{x}-\sum_{q \in Q}\left(D^{q}-\alpha\right) \bar{f}_{j i}^{q}$. Since the right hand side of the latter inequality is a constant for the given LP-solution, finding the most violated two-side inequality on $\operatorname{arc}(i, j)$ for the specific value of $\alpha$ (if one exists) is equivalent to finding the maximal value $\bar{f}_{i j}^{q}$ over all commodities $q \in Q$. This can be done by any sorting algorithm. As for $c$-strong inequalities, computational experiments indicate that two-side inequalities should only be considered for small values of $\alpha$, for instance $\alpha \in\{1,2\}$.

Apart from the inequalities for the edge capacity polytope as described in this paper, we also incorporated some other classes of well-known valid inequalities for network loading problems. Cut-set inequalities are used quite extensively for network loading problems (see for instance Barahona [3], Magnanti et al. [18, 19], Bienstock and Günlük [7], among others). Given a partition of the node set $V$ into two sets $S$ and $T$, let $d[S, T]$ denote the accumulated demand of all commodities with source node in $S$ and sink node in $T$. Then it is clear that the total capacity on the edges in the cut $\delta[S, T]$ should exceed this accumulated demand since all of these commodities must cross the cut. Since, capacity can only be installed in integer amounts, the cut-set inequalities read

$$
\sum_{\{i, j\} \in \delta[S, T]} x_{i j} \geq \max \{\lceil d[S, T]\rceil,\lceil d[T, S]\rceil\} .
$$


Likewise, three partition inequalities (based on a partition of the node set into three sets) have been considered (see [7]), as well as the general $K$-cuts (see Barahona [4]). For small to medium sized graphs as considered in our experiments an exact separation that considers all possible partitions of the graph can be performed reasonably fast, and is therefore used.

\subsection{Computational results edge capacity instances}

To compare the performance of the different ways of separating inequalities for the (undirected) edge capacity polytope, we have generated 20 instances of this single edge problem. For $|Q|=10,25,50,100$ commodities, we constructed 5 instances each with $\lambda=155$ and $d^{q}$ uniformly distributed in the domain $\{10, \ldots, 155\}$. As optimization criterion we minimize $\lambda x-\sum_{q \in Q} c^{q} f^{q}$ with $c^{q}$ a randomly generated integer in the range $\left[0.8 d^{q}, 1.2 d^{q}\right]$ for all $q \in Q$.

Four different strategies are compared: branch-and-bound (B\&B), $c$-strong separation ( $c$-STR), lower convex envelope separation (LCE), and separation of lifted knapsack covers (LKC). As enumeration strategy we selected Best-First-Search with an initial upper bound of 0 .

In Table 1, the results are compared on the number of nodes in the Branch-and-Cut tree, the number of added violated inequalities, and the CPU time. The table shows that

Table 1. Computational results edge capacity instances

\begin{tabular}{|l|l||r|r|r|r||r|r|r||r|r|r|r|}
\hline instance & $|Q|$ & \multicolumn{4}{|c||}{ \# nodes } & \multicolumn{3}{c||}{ \# cuts } & \multicolumn{4}{c|}{ CPU time (sec) } \\
& & B\&B & $c$-STR & LCE & LKC & $c$-STR & LCE & LKC & B\&B & $c$-STR & LCE & LKC \\
\hline \hline ecp010a & 10 & 77 & 11 & 19 & 11 & 16 & 15 & 15 & 0.43 & 0.08 & 0.15 & 0.20 \\
ecp010b & 10 & 43 & 31 & 13 & 19 & 35 & 20 & 31 & 0.36 & 0.30 & 0.17 & 0.36 \\
ecp010c & 10 & 53 & 19 & 15 & 17 & 21 & 17 & 20 & 0.40 & 0.22 & 0.12 & 0.26 \\
ecp010d & 10 & 33 & 13 & 13 & 13 & 10 & 13 & 9 & 0.14 & 0.16 & 0.13 & 0.16 \\
ecp010e & 10 & 103 & 23 & 21 & 21 & 41 & 23 & 24 & 0.63 & 0.25 & 0.22 & 0.28 \\
\hline average & 10 & 61.8 & 19.4 & 16.2 & 16.2 & 24.6 & 17.6 & 19.8 & 0.39 & 0.20 & 0.16 & 0.25 \\
\hline ecp025a & 25 & 149 & 55 & 49 & 55 & 71 & 53 & 74 & 0.80 & 0.68 & 1.44 & 2.26 \\
ecp025b & 25 & 107 & 31 & 47 & 39 & 46 & 61 & 59 & 0.46 & 0.31 & 1.66 & 1.76 \\
ecp025c & 25 & 235 & 87 & 73 & 99 & 127 & 88 & 144 & 1.30 & 1.05 & 2.36 & 4.22 \\
ecp025a & 25 & 131 & 63 & 55 & 49 & 92 & 45 & 42 & 0.79 & 0.80 & 1.19 & 1.43 \\
ecp025e & 25 & 501 & 203 & 135 & 193 & 256 & 126 & 247 & 2.97 & 2.40 & 3.45 & 7.81 \\
\hline average & 25 & 224.6 & 87.8 & 71.8 & 87.0 & 118.4 & 74.6 & 113.2 & 1.26 & 1.05 & 2.02 & 3.50 \\
\hline ecp050a & 50 & 455 & 155 & 157 & 127 & 411 & 124 & 228 & 2.72 & 5.04 & 11.50 & 21.00 \\
ecp050b & 50 & 13 & 41 & 9 & 3 & 93 & 1 & 2 & 0.13 & 1.22 & 0.17 & 0.23 \\
ecp050c & 50 & 353 & 53 & 59 & 53 & 377 & 69 & 75 & 1.97 & 3.23 & 6.28 & 7.25 \\
ecp050d & 50 & 723 & 431 & 99 & 167 & 661 & 115 & 204 & 4.73 & 11.67 & 10.53 & 20.38 \\
ecp050e & 50 & 353 & 147 & 61 & 167 & 271 & 90 & 332 & 2.54 & 4.28 & 7.92 & 30.93 \\
\hline average & 50 & 379.4 & 165.4 & 77.0 & 103.4 & 362.6 & 79.8 & 168.2 & 2.42 & 5.09 & 7.28 & 15.96 \\
\hline ecp100a & 100 & 109 & 69 & 57 & 151 & 125 & 65 & 345 & 0.99 & 5.05 & 22.04 & 116.41 \\
ecp100b & 100 & 79 & 13 & 23 & 13 & 9 & 27 & 9 & 0.62 & 0.58 & 9.10 & 3.51 \\
ecp100c & 100 & 693 & 223 & 273 & 251 & 263 & 279 & 531 & 6.93 & 11.78 & 95.67 & 182.41 \\
ecp100d & 100 & 371 & 197 & 57 & 85 & 241 & 81 & 114 & 3.08 & 10.60 & 27.21 & 42.27 \\
ecp100e & 100 & 1155 & 349 & 271 & 295 & 642 & 425 & 491 & 11.97 & 27.00 & 143.42 & 170.92 \\
\hline average & 100 & 481.4 & 170.2 & 136.2 & 159.0 & 256.0 & 175.4 & 298.0 & 4.72 & 11.00 & 59.49 & 103.10 \\
\hline
\end{tabular}


with separation of lower convex envelope inequalities not only the smallest branch-andcut trees are obtained, but also the number of added cuts is minimized (compared with the other strategies). This indicates that with this strategy very effective inequalities are added. The CPU time of LCE, however, is less attractive (compared with $c$-STR). This is due to the lifting procedure. The lifting procedure for lifted knapsack covers is more complex which explains the CPU times in this case. In most cases the largest number of violated inequalities has been found with the separation of $c$-strong inequalities. It should be mentioned, however, that the results also show that the performance of the different strategies strongly depends on the individual instances. For example, the $c$ strong separation performs badly compared with the other ones on instance ecp $050 \mathrm{~b}$, whereas it outperforms LCE and LKC on the instance ecp 025b in both the number of nodes and cuts. A more detailed investigation of the computations learns that (almost) all lower convex envelope inequalities with slope 1 are also generated with the $c$-strong procedure. Therefore, we propose a combination of $c$-strong separation with separation of the lower convex envelope inequalities for the network loading instances.

\subsection{Computational results network loading instances}

For the network loading problem, we have implemented a branch-and-cut algorithm based on the flow formulation DNFM as described by (1)-(5). The alternative to use a path formulation within the branch-and-cut algorithm has turned out to be less attractive. Although the LP relaxations, even after the addition of valid inequalities, are equivalent, and branching on path variables has some advantages, the exponential growth of the number of path variables is a serious problem for larger graphs. The computational results stated in the sequel are therefore obtained using the flow formulation.

Besides the classes of inequalities related to the edge capacity polytopes, also the already mentioned cut-set inequalities and three partition inequalities are available. To show the importance of both the edge-related inequalities, as well as the cut-related inequalities, we compare 4 different strategies: branch-and-bound (B\&B), separation of edge-related inequalities (EDGE), separation of non-edge-related inequalities (NONEDGE), and separation of all available inequalities (ALL). As discussed in the previous subsection, separation of edge-related inequalities involves the classes of $c$-strong and lower convex envelope inequalities, plus the two-side inequalities. The $c$-strong and lower convex envelope inequalities are separated for both directions of an edge once. For the ALL strategy we consider two variants; with and without an initial upper bound obtained with the heuristics described in [17] (ALL+UB). In all other cases the algorithm starts without an upper bound. Computational experiments show that the DiveAndBest enumeration strategy (Depth-First-Search until a first integer solution is found, Best-First-Search afterwards) performs best for all strategies (in case of an initial upper bound this strategy results in a Best-First-Search).

In Table 2, we compare the quality of the lower bounds derived by the different strategies in the root node of the branch-and-cut tree. The comparison is done for fifteen real-life instances of KPN Research. These instances are defined on complete graphs in the range of 4 to 8 nodes, and for each graph size three different instances with fully dense non-symmetric demand matrices were available. The name of each instance, 
stated in the first column, refers to the number of nodes in the graph (first digit), whereas the second number in the name defines the demand matrix. Besides the lower bounds obtained with the different strategies, the table states the optimal value, and an upper bound obtained with the heuristics described in [17] (used in the second variant of the strategy ALL).

Table 2. Computational results for KPN network loading instances: values

\begin{tabular}{|l|r|r|r|r|r|r|}
\hline Instance & \multicolumn{3}{|c|}{ lower bound root node } & $\begin{array}{r}\text { optimal } \\
\text { value }\end{array}$ & $\begin{array}{r}\text { upper } \\
\text { bound }\end{array}$ \\
& $\begin{array}{r}\text { B\&B } \\
\text { (LP value) }\end{array}$ & EDGE & NONEDGE & ALL & & \\
\hline \hline kpn_4_3 & 1.74 & 2.68 & 3.00 & 3.00 & 3 & 3 \\
kpn_4_10 & 5.81 & 7.17 & 6.75 & 7.19 & 8 & 8 \\
kpn_4_20 & 11.61 & 13.00 & 12.67 & 13.00 & 13 & 13 \\
\hline kpn_5_3 & 1.89 & 3.02 & 3.75 & 3.75 & 4 & 4 \\
kpn_5_10 & 6.32 & 7.98 & 7.67 & 8.50 & 9 & 9 \\
kpn_5_20 & 12.64 & 15.04 & 13.77 & 15.06 & 16 & 16 \\
\hline kpn_6_3 & 1.94 & 3.39 & 4.11 & 4.11 & 5 & 5 \\
kpn_6_10 & 6.45 & 8.07 & 8.11 & 8.36 & 9 & 10 \\
kpn_6_20 & 12.90 & 15.30 & 14.08 & 15.47 & 16 & 17 \\
\hline kpn_7_3 & 1.95 & 3.79 & 4.42 & 4.42 & 6 & 6 \\
kpn_7_10 & 6.52 & 8.35 & 8.54 & 8.81 & 10 & 10 \\
kpn_7_20 & 13.03 & 15.38 & 14.59 & 15.73 & 17 & 18 \\
\hline kpn_8_3 & 2.17 & 4.29 & 4.89 & 4.89 & 7 & 7 \\
kpn_8_10 & 7.22 & 9.44 & 9.74 & 9.83 & 11 & 12 \\
kpn_8_20 & 14.45 & 17.15 & 16.44 & 17.33 & 19 & 21 \\
\hline
\end{tabular}

In Table 3, statistics concerning the number of branch-and-cut nodes, the number of added valid inequalities, and the computation times are reported. The results in both tables show that the network loading problems become very difficult in case the difference between the optimal value and lower bound of the root node is larger than one. This observation holds for all strategies: kpn_5_20 and kpn_6_20 are already too difficult for the NONEDGE strategy, whereas the instance kpn_6_10 cannot be solved with the EDGE strategy. The results of the NONEDGE strategy also indicate that the cut and three-partition inequalities are less powerful in case of larger demands. For the EDGE strategy the relation between the size of the demands and the difficulty of the instance is less clear. In fact, the percentage of the gap closed in the root node by the edge-related inequalities increases as the demand increases, whereas the percentage decreases for the NONEDGE strategy. This observation agrees with our theoretical results that only the fractional part of the demand is important. Another, more surprising, observation that can be made is that not in all cases the separation of more inequalities results in a better overall performance of the algorithm. The instances kpn_5_20, and kpn_6_20 can be solved more efficiently with separation of only the edge-related inequalities than with separation of all inequalities. Also the addition of an initial upper bound does not improve the performance of the algorithm in all cases (see for instance kpn_6_10).

Finally, we have to conclude that for graphs as small as 8 nodes the inequalities are not strong enough to solve the instances to optimality. Reasons for this relatively 
Table 3. Computational results for KPN network loading instances: statistics

\begin{tabular}{|c|c|c|c|c|c|c|c|c|c|c|c|c|c|c|}
\hline \multirow{2}{*}{ Instance } & \multicolumn{5}{|c|}{$\begin{array}{c}\text { \# nodes } \\
\end{array}$} & \multicolumn{4}{|c|}{ \# cuts } & \multicolumn{5}{|c|}{ CPU time (sec) } \\
\hline & $B \& B$ & EDGE & NONEDGE & ALL & ALL+UB & EDGE & NONEDGE & ALL & ALL+UB & B\&B & EDGE & NONEDGE & ALL & $\mathrm{ALL+UB}$ \\
\hline kpn_4_3 & 31 & 5 & 1 & 1 & 1 & 185 & 10 & 34 & 34 & 0.47 & 0.67 & 0.10 & 0.11 & 0.13 \\
\hline kpn_4_10 & 77 & 7 & 47 & 7 & 1 & 99 & 8 & 129 & 35 & $\mid 1.82$ & 0.47 & 1.28 & 0.49 & 0.15 \\
\hline kpn_4_20 & 51 & 1 & 125 & 1 & 1 & 40 & 20 & 54 & 26 & 0.72 & 0.15 & 1.87 & 0.16 & 0.13 \\
\hline kpn_5_3 & 183 & 7 & 5 & 5 & 1 & 478 & 37 & 202 & 74 & 8.95 & 3.76 & 0.48 & 1.16 & 0.34 \\
\hline kpn_5_10 & - & 15 & 3,745 & 5 & 1 & 447 & 1,164 & 262 & 65 & - & 4.52 & 209.36 & 1.90 & 0.36 \\
\hline kpn_5_20 & - & 65 & - & 73 & 1 & 1,097 & - & 1,532 & 156 & - & 16.62 & - & 17.18 & 0.90 \\
\hline kpn_6_3 & - & 43 & 9 & 9 & 1 & 2,879 & 117 & 572 & 147 & - & 90.24 & 2.19 & 7.48 & 1.14 \\
\hline kpn_6_10 & - & - & 1,431 & 11 & 33 & - & 2,043 & 625 & 1,891 & - & - & 240.36 & 10.04 & 33.71 \\
\hline kpn_6_20 & - & 297 & - & 319 & 125 & 6,271 & - & 9,238 & 3,831 & - & 226.01 & - & 238.72 & 89.45 \\
\hline kpn_7_3 & - & - & 27 & 27 & 19 & - & 522 & 1,831 & 1,628 & - & - & 28.56 & 122.83 & 65.90 \\
\hline kpn_7_10 & - & - & - & 693 & 19 & - & - & 19,313 & 1,568 & - & - & - & $1,129.28$ & 64.71 \\
\hline kpn_7_20 & - & - & - & - & 255 & - & - & - & 7,093 & - & - & - & - & 415.06 \\
\hline kpn_8_3 & - & - & - & - & - & - & - & - & - & - & - & - & - & - \\
\hline kpn_8_10 & - & - & - & . & 1 & . & - & - & . & - & - & - & - & - \\
\hline kpn_8_20 & - & - & - & - & - & - & - & & - & - & & - & & \\
\hline
\end{tabular}

bad performance of the branch-and-cut algorithm probably lie in the completeness of the graph (which allows for many different paths) and the unitary cost function which is independent of the edges. For the instance kpn_8_3 the optimal value can be determined by the combinatorial argument that at least 7 links are needed to have a connection between every pair vertices. Since, an upper bound of 7 is also available we can conclude that this is the optimal value. The branch-and-cut algorithms, however, cannot produce a lower bound better than 6 within acceptable time and memory requirements. Also for the other two instances with 8 nodes, the branch-and-cut algorithm is not able to solve them. The optimal values for these instances are obtained by solving a preprocessed version of the linear programming relaxation (with the violated inequalities added in the root node) with the CPLEX integer programming solver. The computation of these optimal values took respectively 3,606 and 163,586 seconds.

\section{Concluding remarks}

In this paper, we have shown that the edge capacity polytope is an important substructure of the network loading problems, both in theory and practice. The computational results of the previous section show that strong valid inequalities increase the value of the LP-relaxation quite substantially. Similar results were obtained by Bienstock and Bley [5], who applied the results of this paper to a multicast network design problem.

Potential gain on our results may lie in extending the approach for a single edge to larger structures such as cuts in the graph. Although cut-set inequalities (or the more general partition inequalities) are facet defining for bifurcated versions of network loading problems (see $[7,19]$ ), for non-bifurcated network loading problems this is in general not the case, and several possibilities for strengthening arise. We have performed some initial computational experiments to test the effect of a strengthening of the cut-set inequalities, but so far the gain has been limited. 


\section{Appendices}

\section{A. $\mathcal{N} \mathcal{P}$-hardness proof of the network loading problem}

We will show $\mathcal{N} \mathcal{P}$-completeness only for the directed case, but from the proof it immediately follows that the undirected case is $\mathcal{N} \mathcal{P}$-hard as well. We need the following problem definitions.

MINIMUM COVER (see Garey and Johnson [10])

INSTANCE: $S=\left\{s_{1}, s_{2}, \ldots, s_{n}\right\}$ and a family $\mathcal{C}$ of subsets of $S$, and an integer $\ell \leq|C|$.

QUESTION: Does there exist a family $\mathcal{C}^{\prime}$ of $\mathcal{C}$ of at most $\ell$ sets such that $\cup_{c^{\prime} \in \mathcal{C}^{\prime}} c^{\prime}=S$ ?

\section{DIRECTED NON-BIFURCATED NETWORK LOADING PROBLEM}

INSTANCE: Graph $G=(V, E)$, a set $Q$ of commodities $\left(s^{q}, t^{q}, d^{q}\right)$, with $d^{q} \in \mathbb{Z}_{0}^{+}$for each $q \in Q$, integer capacity $\lambda$, cost coefficients $c_{i j} \in \mathbb{Z}_{0}^{+}$for every $\{i, j\} \in E$, and a nonnegative integer $L$.

QUESTION: Does there exist a feasible solution for problem $D N F M$ with solution value less than or equal to $L$ ?

Proposition 8. DIRECTED NON-BIFURCATED NETWORK LOADING PROBLEM is strongly $\mathcal{N} \mathcal{P}$-complete.

Proof. It is easy to see that the DIRECTED NON-BIFURCATED NETWORK LOADING PROBLEM is in $\mathcal{N} \mathcal{P}$. Next, given an instance of MINIMUM COVER, construct a graph $G$ as follows. Let $V=S \cup \mathcal{C} \cup\{\operatorname{sink}\}$. Let $\mathcal{C}_{j}$ be an element in $\mathcal{C}$. Introduce an edge $\left\{s_{i}, \mathcal{C}_{j}\right\}$ if $s_{i} \in \mathcal{C}_{j}$, and an edge $\left\{\mathcal{C}_{j}, \operatorname{sink}\right\}$ for all $\mathcal{C}_{j} \in \mathcal{C}$ (see Fig. 2). Next

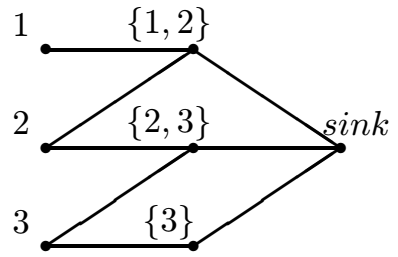

$$
\begin{aligned}
& S=\{1,2,3\} \\
& \mathcal{C}=\{\{1,2\},\{2,3\},\{3\}\}
\end{aligned}
$$

Fig. 2. Transformation of an instance of MINIMUM COVER to DIRECTED NON-BIFURCATED NETWORK LOADING PROBLEM

define a commodity $\left(s_{i}, \sin k, 1\right)$ for each $s_{i} \in S$, and let $\lambda=n$. The capacity costs are defined by $c_{\left\{s_{i}, \mathcal{C}_{j}\right\}}=|\mathcal{C}|+1$ for all $i$ and $j$ and $c_{\left\{\mathcal{C}_{j}, \text { sink }\right\}}=1$ for all $j$. Finally let $L=\ell+|S|(|\mathcal{C}|+1)$. This is clearly a polynomial transformation. We leave it to the reader to verify that a problem instance for MINIMUM COVER with affirmative answer corresponds to an instance with affirmative answer for the DIRECTED NONBIFURCATED NETWORK LOADING PROBLEM, and vice versa. 


\section{B. Proof of Proposition 7: lifting of a valid inequality for the directed edge capacity polytope is $\mathcal{N} \mathcal{P}$-complete}

The proof is a reduction from SUBSET SUM.

SUBSET SUM (cf. Garey and Johnson [10])

INSTANCE: A set of items $A$, a size $s^{q} \in \mathbb{Z}_{0}^{+}$for all $q \in A$, and a positive integer $B$.

QUESTION: Does there exist a subset $\bar{A} \subseteq A$ such that $\sum_{q \in \bar{A}} s^{q}=B$ ?

LIFTING FOR DIRECTED EDGE CAPACITY MODEL

INSTANCE: A set of commodities $Q$, a demand size $d^{q} \in \mathbb{Z}_{0}^{+}$for all $q \in Q$, and a capacity $\lambda \in \mathbb{Z}_{0}^{+}$(this defines an instance of $Y$, using the inequalities $x \geq \sum_{q \in Q} \tilde{d}^{q} f^{q}$ and $x \geq \sum_{q \in Q} \tilde{d}^{q} h^{q}$, where $\left.\tilde{d}^{q}=d^{q} / \lambda\right)$. A complete order $\pi$ on a set of variables $T=\cup_{q \in Q}\left(f^{q} \cup h^{q}\right)$, a specific variable $z \in T$ and an integer $K \in \mathbb{Z}_{0}^{+}$.

QUESTION: If maximal lifting is applied to the inequality $x \geq 0$ in the lifting order $\pi$ to obtain a facet defining inequality for $Y$, is the lifting coefficient of variable $z$ less than or equal to $K$ ?

We show that SUBSET SUM polynomially reduces to LIFTING FOR DIRECTED EDGE CAPACITY MODEL. Given an instance of SUBSET SUM, construct an instance of LIFTING FOR DIRECTED EDGE CAPACITY MODEL as follows. Let $Q=\{1,2,3\} \cup A, \lambda=B$, and define $d^{q}=s^{q}(\lambda+1)$, for all $q \in A$. Define an integer $m=\left[\frac{\sum_{q \in A} d^{q}}{\lambda}\right]$, and let $d^{1}=(m+1) \lambda+1, d^{2}=(m+1) \lambda+\lambda-1, d^{3}=(2 m+4) \lambda+\lambda^{2}$. Define the order $\pi$ on $T$ as $f^{1}, f^{2}, f^{q}, \forall q \in A, h^{3}, h^{2}, h^{1}, h^{q}, \forall q \in A, f^{3}$. Finally, let $z=h^{3}$ and $K=1$.

Next we show that an instance for SUBSET SUM yields an affirmative answer if and only if the corresponding instance for LIFTING FOR DIRECTED EDGE CAPACITY MODEL yields an affirmative answer. For convenience, we will use $\tilde{d}^{q}=d^{q} / \lambda$ throughout the proof. First, note that, starting with $x \geq 0$, after lifting the variables $f^{1}, f^{2}, f^{q}, \forall q \in A$ the valid inequality reads

$$
x \geq b^{T} f=\left\lceil\tilde{d}^{1}\right\rceil f^{1}+\left(\left\lceil\tilde{d}^{2}\right\rceil-1\right) f^{2}+\sum_{q \in A}\left(\left\lceil\tilde{d}^{q}\right\rceil-1\right) f^{q} .
$$

Next, if we apply maximal lifting to variable $h^{3}$ and if $\bar{A} \subseteq A$ is a subset with $\sum_{q \in \bar{A}} s^{q}=B$, then the lifting coefficient $b^{3}$ for the variable $h^{3}$ satisfies

$$
\begin{aligned}
b^{3} & =\min _{(x, f, h) \in Y: h^{3}=1, f^{3}=0, h^{q}=0, \forall q \in Q \backslash\{3\}}\left\{x-\left(b^{T} f\right)\right\} \\
& \leq\lceil\max \{\tilde{d} \hat{q}, \tilde{d}(\{1,2\} \cup \bar{A})\}\rceil-\sum_{q \in\{1,2\} \cup \bar{A}} b^{q} \\
& =2 m+4+\lambda-(2 m+3+\lambda)=1=K .
\end{aligned}
$$

Conversely, let $(\bar{x}, \bar{f}, \bar{h})$ be the vector for which the minimum value less than or equal to $K=1$ in the lifting problem is attained, and let $\bar{Q}=\left\{q \in Q: \bar{f}^{q}=1\right\}$. If $1 \notin \bar{Q}$, then $\sum_{q \in \bar{Q}} \tilde{d}^{q} \leq \tilde{d}^{\hat{q}}$ and hence,

$$
\begin{aligned}
b^{3} & =\min _{(x, f, h) \in Y: h^{3}=1, f^{3}=0, h^{q}=0, \forall q \in Q \backslash\{3\}}\left\{x-\left(b^{T} f\right)\right\} \\
& \geq \tilde{d^{q}}-\left(b^{T} f\right)=2 m+4+\lambda-\left(m+1+\sum_{q \in A} s^{q}\right) \geq 3+\lambda>K
\end{aligned}
$$


since $m \geq \sum_{q \in A} s^{q}$. Hence, $\bar{f}^{1}=1$. Similarly, one can prove that $\bar{f}^{2}=1$. Next define $\tilde{A}=\left\{q \in A: \bar{f}^{q}=1\right\}$, and let $p=\sum_{q \in \tilde{A}} s^{q}$. If $p<B$, then $\tilde{d}(\{1,2\} \cup \tilde{A})<\tilde{d} \tilde{q}^{\hat{q}}$, thus,

$$
b^{3}=\bar{x}-\sum_{q \in \bar{Q}} b^{q}=2 m+4+\lambda-(2 m+3+p)=\lambda-p+1>K
$$

so, $p<B$ cannot be the case. If $p>B$, then $\tilde{d}(\{1,2\} \cup \tilde{A})>\tilde{d} \hat{q}$, hence,

$$
b^{3}=\bar{x}-\sum_{q \in \bar{Q}} b^{q}=2 m+3+p+\left\lceil\frac{p}{\lambda}\right\rceil-(2 m+3+p)=\left\lceil\frac{p}{\lambda}\right\rceil \geq 2>K
$$

hence, neither $p>B$ can be the case. But this yields that $\sum_{q \in \tilde{A}} s^{q}=p=B$, hence $\tilde{A}$ is the required subset.

\section{References}

1. Atamturk, A., Rajan, D. (2001): On splittable and unsplittable capacitated network design arc-set polyhedra. To appear in Mathematical Programming

2. Balas, E. (1975): Facets of the knapsack polytope. Mathematical Programming 8, 146-164

3. Barahona, F. (1996): Network design using cut inequalities. SIAM Journal on Optimization 6, 823-837

4. Barahona, F. (2000): On the $k$-cut problem. Operations Research Letters 26(3), 99-105

5. Bienstock, D., Bley, A. (2000): Proceedings in the 8th International Conference on Telecommunication Systems, March 9-12, 2000. Nashville

6. Bienstock, D., Chopra, S., Günlük, O., Tsai, C.-Y. (1998): Minimum cost capacity installation for multicommodity network flows. Mathematical Programming 81, 177-199, July 1998. Working paper

7. Bienstock, D., Günlük, O. (1996): Capacitated network design - polyhedral structure and computation. ORSA Journal on Computing 8, 243-260

8. Brockmüller, B., Günlük, O., Wolsey, L.A. (1996): Designing private line networks - polyhedral analysis and computation. Discussion Paper 9647, Center for Operations Research and Econometrics, October 1996

9. Brockmüller, B., Günlük, O., Wolsey, L.A. (1998): Designing private line networks - polyhedral analysis and computation. Discussion Paper 9647 revised, Center for Operations Research and Econometrics, March 1998

10. Garey, M.R., Johnson, D.S. (1979): Computers and intractability: a guide to the Theory of $\mathcal{N} \mathcal{P}$-Completeness. Freeman and Company, N.Y.

11. Gavish, B., Altinkemer, K. (1990): Backbone network design tools with economic tradeoffs. ORSA Journal on Computing 2(3), 236-252

12. Gu, Z., Nemhauser, G.L., Savelsbergh, M.W.P. (1998): Lifted cover inequalities for 0-1 integer programs: Complexity. INFORMS Journal on Computing 11, 117-123

13. Gu, Z., Nemhauser, G.L., Savelsbergh, M.W.P. (1998): Lifted cover inequalities for 0-1 integer programs: Computation. INFORMS Journal on Computing 10, 427-437

14. Hammer, P.L., Johnson, E.L., Peled, U.N. (1975): Facets of regular 0-1 polytopes. Mathematical Programming 8, 179-206

15. van Hoesel, C.P.M., Koster, A.M.C.A., van de Leensel, R.L.M.J., Savelsbergh, M.W.P. (2000): Polyhedral results for the edge capacity polytope. Technical Report ZIB 00-22, Konrad-Zuse-Zentrum für Informationstechnik Berlin, Berlin, Germany. Available at http://www.zib.de/koster/

16. CPLEX division of ILOG. CPLEX callable library, version 6.5, 1999

17. van de Leensel, R.L.M.J. (1999): Models and Algorithms for Telecommunication Network Design. PhD thesis, Maastricht University

18. Magnanti, T.L., Mirchandani, P., Vachani, R. (1993): The convex hull of two core capacitated network design problems. Mathematical Programming 60, 223-250

19. Magnanti, T.L., Mirchandani, P., Vachani, R. (1995): Modelling and solving the two-facility capacitated network loading problem. Operations Research 43, 142-157

20. Marchand, H., Wolsey, L.A. (1999): The 0-1 knapsack problem with a single continous variable. Math. Program. 85, 15-33 
21. Padberg, M. (1975): A note on zero-one programming. Operations Research 23, 833-837

22. Thienel, S. (1998): ABACUS: A Branch And CUt System, version 2.2

23. Wessäly, R. (2000): DImensioning Survivable Capacitated NETworks. PhD thesis, TU Berlin, Fakultät für Mathematik, Berlin, Germany, April 2000

24. Wolsey, L.A. (1975): Faces of linear inequalities in 0-1 variables. Mathematical Programming 8, 165-178

25. Wolsey, L.A. (1976): Facets and strong inequalities for integer programs. Operations Research 24, 367372 